\title{
COVID-19: Implications, Reactions and Future Directions
}

\author{
Review Rahim Dhanani ${ }^{1}$ (1), Muhammad Wasif ${ }^{2}$ (D), Hamdan Ahmed Pasha ${ }^{3}$ (D), Muntazir Hussain ${ }^{4}$ (D), \\ Shayan Khalid Ghaloo ${ }^{2}$ (D), Abdul Basit Shah Vardag ${ }^{2}$ (D), Khadija Mahmood ${ }^{5}$ (D), Raza Hussain ${ }^{1}$ (1) \\ ${ }^{1}$ Department of Surgical Oncology, Shaukat Khanum Memorial Cancer Hospital \& Research Center, Lahore, Pakistan \\ ${ }^{2}$ Department of Otolaryngology, Head and Neck Surgery, Aga Khan University Hospital, Karachi, Pakistan \\ ${ }^{3}$ Department of Otolaryngology Head and Neck Surgery, Jinnah Medical College Hospital, Karachi, Pakistan \\ ${ }^{4}$ Clinic of Head and Neck Surgery, Cancer Foundation Hospital, Karachi, Pakistan \\ ${ }^{5}$ Clinic of Medicine, Abbasi Shaheed Hospital, Karachi, Pakistan
}

Abstract

ORCID iDs of the authors: R.D. 0000-0002-5519-862x; M.W. 0000-0002-3760-484x; H.A.P. 0000-0002-6620-7544; M.H. 0000-0002-5688-1949; S.K.G. 0000-0002-3237-0669; A.B.S.V. $0000-0001-8257-219 x$ K.M. 0000-0001-8926-2813; R.H. 0000-0002-3552-722X.

Cite this article as: Dhanani R, Wasif $M$, Pasha HA, Hussain M, Ghaloo SK, Vardag, AB, et al. COVID-19: Implications, Reactions and Future Directions. Turk Arch Otorhinolaryngol 2020; 58(2): 122-6.

\section{Corresponding Author:}

Muhammad Wasif, wasif_siddiq313@yahoo.com

Received Date: 02.05 .2020 Accepted Date: 03.06.2020

Content of this journal is licensed under a Creative Commons Attribution 4.0 International License. Available online at www.turkarchotolaryngol.net
Humanity has faced several foes over the centuries, a formidable one amongst them is the current pandemic of COVID-19. The symptoms of COVID-19 are more or less related to the nose and throat. Therefore, patients more often present to Ear Nose Throat (ENT) clinics with symptoms including cough, sore throat, fever and shortness of breath. In the management of head and neck pathologies, as the airway is a direct source of infection, the impact of COVID-19 holds special significance. This review has attempted to explain the various aspects of the disease itself, its diagnosis, the use of personal protective equipment (PPE) to provide an overview of the evolving recommendations in head and neck patients, the future outlook and the limitations faced in developing countries specifically for ENT patients.

Keywords: COVID-19, otorhinolaryngology, pandemics, elective surgical procedures

\section{Introduction}

Humanity has faced several foes over the centuries, a formidable one amongst them is the current pandemic of Corona virus disease of 2019 (COVID-19). It is believed that it originated in Wuhan, the capital city of Hubei province in the People's Republic of China, COVID-19 was labelled as a public health emergency of international significance by World Health Organization (WHO) in January 2020 (1). The issue has raised hurdles in healthcare provision in all medical sectors and has been dealt with in different ways. The pandemic is a stark reminder of the emerging trends in infectious diseases and the need for the constant evolution of healthcare.

In the management of head and neck pathologies, as the airway is a direct source of infection, the impact of COVID-19 holds special significance (2). This review article has attempted to explain the various aspects of the disease itself, providing an overview of the evolving recommendations for head and neck patients, the future outlook and the limitations faced in developing countries, specifically for otorhinolaryngology patients.

\section{What Is COVID-19?}

\section{A brief history of Coronavirus}

In the past two decades, there have been two catastrophic events where the Coronavirus crossing over from animal to humans has resulted in severe disease. In 2002-2003, a new coronavirus of $\beta$-genera originating from bats and crossing over to humans via cats was designated as severe acute respiratory syndrome coronavirus (SARS-CoV). This virus affected 8,422 people, mostly in Guangdong province of China and some parts of Hong Kong and caused more than 900 deaths with a mortality rate of $11 \%$ (3). A decade later, another virus of bat origin emerged in Saudi Arabia in 2012 and was named as the Middle East respiratory syndrome coronavirus (MERS-CoV). Dromedary camels acted as the intermediate host and up to 2,500 people were affected, out of whom 858 lost their lives with a fatality rate of $34 \%$ (4).

\section{Viral etiology}

According to the recent literature, SARS-CoV-2 resembles SARS-CoV and MERS-CoV. Being 
zoonotic, its probable origin is from horseshoe bats and pangolins acting as the most likely intermediate hosts $(5,6)$.

\section{Epidemiological considerations}

Mode of transmission: on the basis of epidemiological research and the data available, it seems that the COVID-19 outbreak initially began from an animal to the human transmission which then led to human to human spread (7). It is now suggested that this interpersonal spread is due to contact transmission and via respiratory droplets (8). Studies have found SARS-CoV-2 in the stool of few patients with COVID-19 in China and the United States, and it is considered that there may be a risk of fecal-oral transmission as well (7). Although the spread of this virus through aerosols and from mothers to newborn need yet to be confirmed $(9,10)$.

Source of transmission: The main sources of transmission of the disease are those individuals who have tested positive for COVID-19. Other than that, recent studies and observations suggested that asymptomatic patients and those in their incubation period also act as carriers of SARS-CoV-2 and transmit the disease. It is questionable whether or not patients in the recovery phase are source of transmission $(5,11)$.

Incubation period: The estimated incubation period of COVID-19 is 5 to 6 days on the average. This can, however, go up to 14 days, which, therefore, is adopted as the observation and quarantine period for exposed persons (7). Few studies in the literature also reported incubation periods up to 24 days (12).

Mortality rate: Current data suggests that mortality rate of COVID-19 is $0.39 \%$ to $4.05 \%$ and comparatively lower than those of SARS (mortality rate up to 10\%) and MERS (mortality rate up to $34 \%$ ). It is also higher than the mortality rate of seasonal influenza, which accounts for up to $0.01 \%$ to $0.17 \%$ (7).

People at high risk: People of all ages are found to be susceptible to this disease. Although people who have close contact with patients infected with COVID-19, including healthcare professionals and other patients admitted in the hospital are at higher risk (13). People with immunosuppression, multiple comorbidities and old age are considered to be at risk to become victims of this infection.

\section{Main Points}

- The symptoms of COVID-19 are more or less related to the nose and throat including cough, sore throat, fever, anosmia, dysgeusia and shortness of breath.

- Hospitalized patients may require intensive care support and can develop complications such as acute respiratory distress syndrome, arrhythmia, shock, acute kidney injury, ultimately leading to multi-organ failure and death.

- PPEs reduce risk of nosocomial infections, AAOHNS advocated adherence to respiratory droplet precautions by using eye protection, gown, gloves and masks (N95 for procedures).

- Guidelines are being updated on almost daily basis, proper use of PPE and a more stringent selection of patients for examination and surgical procedures the risk can be minimized.

\section{Clinical manifestations and complications}

Infection is slightly more male predominant with a rate ranging around $58 \%$ (14). The symptoms of COVID-19 are more or less related to the nose and throat. Therefore, patients more often present to Ear Nose Throat (ENT) clinics with symptoms including cough, sore throat, fever and shortness of breath. Other atypical symptoms can include fatigue, headache, diarrhea, vomiting and confusion (13). Since some patients from China, Italy and Iran have reported anosmia and dysgeusia, these should also be considered when screening patients for COVID-19 (15).

One-fourth to one-third of hospitalized patients may require intensive care support and can develop complications such as acute respiratory distress syndrome, arrhythmia, shock, acute kidney injury and acute liver disease, ultimately leading to the vicious cycle of multi-organ failure and death (7).

\section{Diagnosis and treatment}

Laboratory workup may show raised C-reactive protein (CRP), lactate dehydrogenase (LDH) and erythrocyte sedimentation rate (ESR) levels along with lymphocytopenia (16). Since chest $\mathrm{X}$-ray and chest computed tomography $(\mathrm{CT})$ show nonspecific findings, the American College of Radiology (ACR) and the Society of Thoracic Radiologists recommend that these techniques are not used for screening or for diagnostic purposes. ACR recommended computed tomography $(\mathrm{CT})$ should not be used as first-line test to diagnose this condition but should be reserved for hospitalized and symptomatic patients and appropriate infection control measures should be taken before scanning subsequent patients. Furthermore, ACR suggested that a normal CT does not rule out COVID-19 infection and an abnormal CT is not specific for COVID-19 diagnosis (2).

Currently, the diagnosis of COVID-19 is made with polymerase chain reaction (PCR) testing for nucleic acid sequence homology in nasopharyngeal or throat swabs. The preferred site is the nasopharynx because of the higher viral load (17). The range of sensitivity of the PCR test reported in literature is from $30 \%$ to $60 \%$. This low sensitivity may be due to multiple external factors including inappropriate sampling technique or collection, transportation and quality or performance of the kits (18). Single negative PCR test result from a suspected COVID-19 patient does not exclude the infection and may become positive on repeating the test, and therefore we should be alert of the patients with COVID-19 related symptoms and suspected history (2). Serological testing for COVID-19 is currently under development (19).

In a video, Marty et al. (20) demonstrated the proper technique of obtaining specimens from the surface of the respiratory $\mathrm{mu}^{-}$ cosa with nasopharyngeal swabs for the diagnosis of COVID-19 in adults and children.

Until now, there is no specific treatment available against this infection, and therefore the current management of COVID-19 is to control the source of infection with the help of infection control measures to decrease the risk of its transmission and provision of early diagnosis, isolation and supportive care (7). 


\section{Infection Control}

\section{Risk of nosocomial infection}

The risk of nosocomial infection stems from multiple factors: at the start of the pandemic, healthcare personnel did not have adequate knowledge about the nature of the spread of the virus and that led to incomplete protective measures, the increasing workload led to fatigue, which in turn increased the probability of acquiring the infection, and later in the course of the pandemic shortage of personal protective equipment (PPE) itself became a grave concern (21).

\section{Infection control guidelines and protocols}

Various institutions introduced protocols to control infection spread amongst healthcare workers (HCW). One Chinese hospital divided the strategy into branches like material allocation, infection control training, a triage strategy, reducing hospital traffic, and a cleaner environment. They kept waiting-areas apart, reduced appointments, relieved HCW $>65$ years of age and attended only to emergencies. Surgical masks were made compulsory on floors, and repeated temperature checks of patients were done. They restricted patient visitors to one and for suspected patients they made an emergency isolation ward. Surgeries that were elective in nature were initially put on hold until control measures were established. These surgeries were then started gradually to ensure familiarity with the new systems. With these measures, they reported that they had no case of nosocomial infection in their hospital (22).

More specific stringent measures were taken by the American Academy of Otolaryngology-Head and Neck Surgery (AAOHNS) (23) that included cancellation of all elective cases, converting all tracheotomies to percutaneous routes, keeping patients intubated for up to three weeks, operating in negative pressure operating room $(\mathrm{OR})$, limiting the $\mathrm{OR}$ personnel with teams involved in cases of suspected COVID patients. All endoscopic or skull base procedures were abandoned and all consults for laryngoscopy, nasopharyngoscopy, nasal endoscopy, were suspended unless needed in urgent/emergent situation. All clinic patients had their status rescheduled or offered telemedicine visits.

\section{Use of personal protective equipment (PPE)}

PPEs reduce the risk of nosocomial infections, as seen during the SARS outbreak (24). Thus, it was applied to mitigate the COVID-19 spread. Previous studies proved masks a significant source of contamination and otolaryngologists need to be adept with proper procedures and cautions when removing or re-using masks (25). However, a recent systematic review meta-analyzed data from four randomized control trials (RCT) to conclude that "Low certainty evidence suggests that medical masks and not resistant to oil (N95) respirators offer similar protection against viral respiratory infection including coronavirus in healthcare workers during non-aerosol-generating care." They recommended N95 masks to be preserved for only aerosol-generating procedures (26).
The AAOHNS produced guidelines for the proper use of PPE. They advocated adherence to respiratory droplet precautions by using eye protection, gown, gloves and masks (N95 for procedures), practice donning and removing PPE, confirm visibility, maintain communication, assess fidelity to perform safe endoscopy and finally being alert to the possibility of asymptomatic COVID-19 infection (23).

\section{Recommendations for Otolaryngologists/Head and Neck Surgeons}

\section{ENT/head and neck examination}

Examination of patients presenting to otolaryngology clinic with routine complaints is a challenge in the era of this pandemic. Public Health England (PHE) advised ENT physicians to wear full PPE including N95 masks, gloves, gown and face shield or goggles for all type of examinations or procedures in all patients. They further suggested that for conditions not involving the oral cavity such as neck and skin lesions, routine COVID PPE including gloves, surgical mask and apron could be used (27).

\section{Procedural considerations}

Majority of the procedures in otolaryngology, especially oral, nasal and airway surgeries put the surgeon at a higher risk of contracting the disease. Here we briefly discuss the most important procedures.

\section{Role of tracheotomies}

A major complication of COVID 19 is acute respiratory distress syndrome (ARDS) which requires mechanical ventilation, and the timing and the need for tracheotomy in these patients is a very pertinent question under the current conditions. In a small sample of 28 patients, Mattioli et al. (28) reported no difference between percutaneous and surgical tracheotomy in terms of timing and the number of affected team members; they also suggested to do the tracheotomy on $7^{\text {th }}$ to $14^{\text {th }}$ days in selected patients. Guidelines issued by the Ear Nose Throat United Kingdom (ENT UK) recommends COVID 19 testing in all patients planned for tracheotomy. They further recommended using cuffed non-fenestrated tracheotomy tubes, not piercing the cuff of the endotracheal tube while performing tracheotomy and ceasing ventilation while the window in the trachea is being performed and prior to inserting the tracheostomy tube (29). Full PPE including gown, gloves, N95 mask, face shield should be used while performing the procedure.

\section{Endoscopic sinus surgery}

The viral load of COVID-19 is highest in the nasal cavity regardless of whether the patient is symptomatic or asymptomatic (17). A case operated on in Wuhan, China for trans-nasal pituitary adenoma resulted in affecting more than 14 healthcare workers who were involved in that surgery (30). Due to this high potential for infectivity, we recommend that all cases of non-emergent nature should be deferred indefinitely until more data is available on this topic. In case of emergency, all necessary precautions should be observed. The number of people in 
the OR should be limited to only the necessary individuals, the procedure should be performed by the consultant surgeon and not by trainees to limit the time of exposure and all personnel involved in the case should use proper PPEs including gowns, gloves, N95 masks, face shields.

\section{Ear surgery}

Whether COVID-19 can involve the respiratory mucosa lining of the middle ear and the mastoid air cells is not known. Since COVID-19 can involve the rest of the airway mucosa, it is highly likely that it can also involve and infect the lining of the eustachian tube, the middle ear and the mastoid cells.

Drilling the mastoid bone creates droplets and aerosols, and in the presence of the virus, this procedure can cause all individuals in operating room to be infected. Mastoidectomy is therefore considered a high-risk procedure. It is important to take all the necessary precautions and operate on only in emergency cases such as those with complex chronic ear conditions. Ideally, preoperative testing for COVID-19 should be performed in all patients undergoing ear surgery. Surgery should be deferred until the patient is disease free in case patient's test is positive for COVID-19 (31).

\section{The Role of Head and Neck Surgeon During the COVID-19 Pandemic}

Head and neck patients are known to practice self-referral (32). Hence it is essential to run outpatient services at times when the presentation of these patients to the emergency department would lead to increased demand on an already over-burdened department catering to patients of COVID-19.

Head and neck surgeons, together with anesthesiologists, are responsible for airway management. As more and more intensive care staff are exposed to the disease, ENT doctors will be crucial in the airway management of COVID-19 patients.

As the pandemic continues, there will be a rise in the number of patients that require prolonged mechanical ventilation, hence an increase in the number of tracheotomies performed. The mean duration of viral shedding is 20 days (33). Tracheotomy produces aerosol and is a high-risk procedure in these patients (29).

$\mathrm{ENT}$, as is the rest of medicine, will face a lot of challenges in the few weeks to come. Let's stay healthy, so we can help our patients.

\section{Challenges Faced by Developing Countries}

Many developing countries have been pushed into crisis after the rapid spread of the novel coronavirus. Implementation of countrywide lockdowns and standard operating procedures have turned into great challenges in developing countries more than in developed countries.

\section{Health-related challenges}

The expenditure of funds on healthcare systems in developing countries is far less to enjoy better health services. Nearly all healthcare systems worldwide are coping with problems of high costs and lack of facilities.
Developing countries have the lowest number of intensive care units (ICU) per capita. The reason behind this is the cost of trained healthcare workers, infrastructure, and supplies that are required to establish a standard ICU (34).

People are devoid of the basic necessities that are essential for prevention, including facemasks, gloves and disinfectants. With the increasing number of cases, hospitals are facing a shortage of testing kits and unable to perform mass screening (35).

\section{Social challenges}

A major amount of population in developing countries is living in slums. In Mumbai, India, which is a city with a total surface area of 603 kilometers squared, has an estimated population of over 22 million that has doubled since 1991. The percentage of people living in slums is estimated to be as high as $41.3 \%$ (36). The population in such areas is so dense, and streets are not wide enough to follow the social distancing guidelines. This makes such areas a breeding ground for the virus. Many people do not have access to clean drinking water, they have to travel daily to fetch water from a local community water supply, where human interaction is not a choice but a compulsion.

\section{Economic challenges}

Most of the people live hand-to-mouth on daily wages and cannot afford to stock up on food. So, they are faced with the dilemma of either going out to work and risk being contaminated or staying at home and face extreme hunger. Once the lockdown is implemented, taking care of such people becomes a responsibility of the state. And the states of developing countries in such cases become handicapped.

\section{Conclusion}

COVID-19 has presented humanity with unprecedented challenges. Healthcare professionals are at considerably higher risk due to the close contact with these patients. Otorhinolaryngologists are at greater risk as the viral load is greatest in the nasopharynx and oral cavity. The evidence is limited and guidelines are being updated on almost daily basis. With proper use of $\mathrm{PPE}$ and a more stringent selection of patients for examination and surgical procedures the risk can be minimized.

Peer-review: Externally peer-reviewed.

Author Contributions: Concept - R.D.; Supervision - R.H.; Writing - M.W., H.A.P., M.H., S.K.G., A.B.S.V.; Critical Reviews - K.M.

Conflict of Interest: The authors have no conflicts of interest to declare.

Financial Disclosure: The authors declared that this study has received no financial support.

\section{References}

1. Jotz GP, Voegels RL, Bento RF. Otorhinolaryngologists and Coronavirus disease 2019 (COVID-19). Int Arch Otorhinolaryngol 2020; 24: 125-8. [Crossref]

2. Vukkadala N, Qian ZJ, Holsinger FC, Patel ZM, Rosenthal E. COVID-19 and the otolaryngologist: preliminary evidence-based 
review. Laryngoscope 2020 Mar 26. doi: 10.1002/lary.28672. [Epub ahead of print]. [Crossref]

3. Chan-Yeung M, Xu RH. SARS: epidemiology. Respirology 2003: 8: 9-14. [Crossref]

4. Memish ZA, Perlman S, Van Kerkhove MD, Zumla A. Middle East respiratory syndrome. Lancet 2020; 395: 1063-77. [Crossref]

5. Chan JFW, Yuan S, Kok KH, To KKW, Chu H, Yang J, et al. A familial cluster of pneumonia associated with the 2019 novel coronavirus indicating person-to-person transmission: a study of a family cluster. Lancet 2020; 395: 514-23. [Crossref]

6. Lu R, Zhao X, Li J, Niu P, Yang B, Wu H, et al. Genomic characterization and epidemiology of 2019 novel coronavirus: implications for virus origins and receptor binding. Lancet 2020; 395: 565-74. [Crossref]

7. Meng L, Hua F, Bian Z. Coronavirus disease 2019 (COVID-19): Emerging and future challenges for dental and oral medicine. J Dent Res 2020: 99: 481-7. [Crossref]

8. Association CPM. [An update on the epidemiological characteristics of novel coronavirus pneumonia (COVID-19)]. Chin J Epidemiol 2020; 41: 139-44.

9. Chen H, Guo J, Wang C, Luo F, Yu X, Zhang W, et al. Clinical characteristics and intrauterine vertical transmission potential of COVID-19 infection in nine pregnant women: A retrospective review of medical records. Lancet 2020; 395: 809-15. [Crossref]

10. Zhu H, Wang L, Fang C, Peng S, Zhang L, Chang G, et al. Clinical analysis of 10 neonates born to mothers with $2019-\mathrm{nCoV}$ pneumonia. Transl Pediatr 2020; 9: 51-60. [Crossref]

11. Rothe C, Schunk M, Sothmann P, Bretzel G, Froeschl G, Wallrauch $\mathrm{C}$, et al. Transmission of 2019-nCoV infection from an asymptomatic contact in Germany. N Engl J Med 2020; 382: 970-1. [Crossref]

12. Bai Y, Yao L, Wei T, Tian F, Jin DY, Chen L, et al. Presumed asymptomatic carrier transmission of COVID-19. JAMA 2020; 323: 1406-7. [Crossref]

13. Wang D, Hu B, Hu C, Zhu F, Liu X, Zhang J, et al. Clinical characteristics of 138 hospitalized patients with 2019 novel coronavirus-infected pneumonia in Wuhan, China.JAMA 2020; 323: 1061-9. [Crossref]

14. Guan WJ, Ni ZY, Hu Y, Liang WH, Ou CQ, He JX, et al. Clinical characteristics of coronavirus disease 2019 in China. N Engl J Med 2020; 382: 1708-20. [Crossref]

15. Hopkins C, Kumar N. Loss of sense of smell as marker of COVID-19 infection. ENT UK 2020. (2020 Mar 26) Available from: https://www entuk org/sites/default/files/files/Loss of sense of smell as marker of COVID.pdf.

16. Lippi G, Plebani M. Procalcitonin in patients with severe coronavirus disease 2019 (COVID-19): A meta-analysis. Clin Chim Acta 2020; 505: 190-1. [Crossref]

17. Zou L, Ruan F, Huang M, Liang L, Huang H, Hong Z, et al. SARS-CoV-2 viral load in upper respiratory specimens of infected patients. N Engl J Med 2020; 382: 1177-9. [Crossref]

18. Ai T, Yang Z, Hou H,Zhan C, Chen C, LvW, et al. Correlation of chest CT and RT-PCR testing in coronavirus disease 2019 (COVID-19) in China: A report of 1014 cases. Radiology 2020 Feb 26. doi: 10.1148/ radiol.2020200642. [Epub ahead of print]. [Crossref]

19. Zhang W, Du RH, Li B, Zheng XS, Yang XL, Hu B, et al. Molecular and serological investigation of 2019-nCoV infected patients: implication of multiple shedding routes. Emerg Microbes Infect 2020; 9: 386-9. [Crossref]

20. Marty FM, Chen K, Verrill KA. How to obtain a nasopharyngeal swab specimen. N Engl J Med 2020; 382: 76. [Crossref]
21. Wang J, Zhou M, Liu F. Reasons for healthcare workers becoming infected with novel coronavirus disease 2019 (COVID-19) in China. J Hosp Infect 2020; 105: 100-1. [Crossref]

22. Lu D, Wang H, Yu R, Yang H, Zhao Y. Integrated infection control strategy to minimize nosocomial infection of coronavirus disease 2019 among ENT healthcare workers. J Hosp Infect 2020; 104: 454-5. [Crossref]

23. Balakrishnan K, Schechtman S, Hogikyan ND, Teoh AYB, McGrath B, Brenner MJ. COVID-19 pandemic: What every otolaryngologisthead and neck surgeon needs to know for safe airway management. Otolaryngol Head Neck Surg 2020; 162: 804-8. [Crossref]

24. Seto WH, Tsang D, Yung RWH, Ching TY, Ng TK, Ho M, et al. Effectiveness of precautions against droplets and contact in prevention of nosocomial transmission of severe acute respiratory syndrome (SARS). Lancet 2003; 361: 1519-20. [Crossref]

25. Suen LKP, Guo YP, Tong DWK, Leung PHM, Lung D, Ng MSP, et al. Self-contamination during doffing of personal protective equipment by healthcare workers to prevent Ebola transmission. Antimicrob Resist Infect Control 2018; 7: 157. [Crossref]

26. Bartoszko JJ, Farooqi MAM, Alhazzani W, Loeb M. Medical masks vs N95 respirators for preventing COVID-19 in healthcare workers a systematic review and meta-analysis of randomized trials. Influenza Other Respir Viruses 2020; 14: 365-73. [Crossref]

27. OMFS and ENT surgeons issue new COVID-19 PPE guidance. Br Dent J 2020; 228: 571. [Crossref]

28. Mattioli F, Fermi M, Ghirelli M, Molteni G, Sgarbi N, Bertellini E, et al. Tracheostomy in the COVID-19 pandemic. Eur Arch Otorhinolaryngol 2020; 277: 2133-5. [Crossref]

29. Harrison L, Ramsden J, Winter S, Rocke J, Heward E. Guidance for surgical tracheostomy and tracheostomy tube change during the COVID-19 pandemic. ENT UK 2020 (2020 Mar 19). Available from: https://www.entuk.org/tracheostomy-guidance-during-covid-19-pandemic.

30. Patel ZM, Fernandez-Miranda J, Hwang PH, Nayak JV, Dodd R, Sajjadi H, et al. Precautions for endoscopic transnasal skull base surgery during the COVID-19 pandemic. Neurosurgery 2020; 87: E66-E67. [Crossref]

31. Givi B, Schiff BA, Chinn SB, Clayburgh D, Iyer NG, Jalisi S, et al. Safety recommendations for evaluation and surgery of the head and neck during the COVID-19 pandemic. JAMA Otolaryngol Head Neck Surg 2020 Mar 31. doi: 10.1001/jamaoto.2020.0780. [Epub ahead of print]. [Crossref]

32. Naunheim MR, Kozin ED, Sethi RK, Ota HG, Gray ST, Shrime MG. Contingent valuation analysis of an otolaryngology and ophthalmology emergency department: The value of acute specialty care. JAMA Otolaryngol Head Neck Surg 2017; 143: 234-8. [Crossref]

33. Zhou F, Yu T, Du R, Fan G, Liu Y, Liu Z, et al. Clinical course and risk factors for mortality of adult inpatients with COVID-19 in Wuhan, China: A retrospective cohort study. Lancet 2020; 395: 1054-62. [Crossref]

34. Dünser MW, Baelani I, Ganbold L. A review and analysis of intensive care medicine in the least developed countries. Crit Care Med 2006; 34: 1234-42. [Crossref]

35. Daw MA. Corona virus infection in Syria, Libya and Yemen; an alarming devastating threat. Travel Med Infect Dis 2020 Apr 2. doi: 10.1016/j.tmaid.2020.101652. [Epub ahead of print]. [Crossref]

36. World Population Review. Mumbai Population 2020. Available from: https://worldpopulationreview.com/world-cities/mumbai-population/. 